\title{
BMJ Open Skeletal muscle function and need for long-term care of urban elderly people in Japan (the Bunkyo Health Study): a prospective cohort study
}

\author{
Yuki Someya, ${ }^{1,2,3}$ Yoshifumi Tamura, ${ }^{1,2}$ Hideyoshi Kaga, ${ }^{2}$ Shuko Nojiri, ${ }^{4}$ \\ Kazunori Shimada, ${ }^{1,5}$ Hiroyuki Daida, ${ }^{1,5}$ Muneaki Ishijima, ${ }^{1,6}$ Kazuo Kaneko, ,,6 \\ Shigeki Aoki, ${ }^{1,7}$ Takashi Miida, ${ }^{1,8}$ Satoshi Hirayama, ${ }^{1,8}$ Seiki Konishi, ${ }^{1,8}$ \\ Nobutaka Hattori, ${ }^{1,9}$ Yumiko Motoi, ${ }^{1,10}$ Hisashi Naito, ${ }^{1,3}$ Ryuzo Kawamori, ${ }^{1,2}$ \\ Hirotaka Watada ${ }^{1,2}$
}

To cite: Someya Y, Tamura Y, Kaga $\mathrm{H}$, et al. Skeletal muscle function and need for longterm care of urban elderly people in Japan (the Bunkyo Health Study): a prospective cohort study. BMJ Open 2019;9:e031584. doi:10.1136 bmjopen-2019-031584

- Prepublication history for this paper is available online. To view these files please visit the journal online (http://dx.doi. org/10.1136/bmjopen-2019031584).

Received 10 May 2019 Revised 17 August 2019 Accepted 20 August 2019
Check for updates

(C) Author(s) (or their employer(s)) 2019. Re-use permitted under CC BY-NC. No commercial re-use. See rights and permissions. Published by BMJ.

For numbered affiliations see end of article.

Correspondence to Dr Yoshifumi Tamura; ys-tamur@juntendo.ac.jp

\section{ABSTRACT}

Purpose The proportion of elderly individuals (age $\geq 65$ years) in Japan reached $27.7 \%$ in 2017 , the highest in the world. A serious social problem in a super-aged society is the rise in the number of elderly people who need longterm care (LTC), which is mainly due to cerebrovascular disease, dementia, age-related frailty, falls and fractures, and joint disease. We hypothesised that decreased muscle mass, muscle strength and insulin sensitivity are the common risk factors for these diseases related to needing LTC. We developed a prospective cohort study of elderly subjects in an urban community to test this hypothesis. The primary objective is to prospectively investigate associations between muscle mass, muscle strength, and insulin sensitivity and incidence of main disease and risk factors of needing LTC. The primary outcomes are the incidence of cerebrovascular disease and cognitive decline.

Participants Participants were 1629 people aged 65-84 years living in 13 communities in an urban area (Bunkyo-ku, Tokyo, Japan). Average age was 73.1 \pm 5.4 years.

Findings to date We obtained baseline data on cognitive function, cerebral small vessel disease (SVD) determined by brain MRI, body composition, bone mineral density, arteriosclerosis, physical function, muscle mass, muscle strength and insulin sensitivity. Mild cognitive impairment and dementia were observed in $18.1 \%$ and $3.3 \%$ of participants, respectively. The prevalence of cerebral SVD was $24.8 \%$. These characteristics are similar to those previously reported in elderly Japanese subjects.

Future plans We will ask participants about their health status, including incidence of cerebrovascular disease, falls, fractures and other diseases every year by mail. We plan to re-evaluate cognitive function, brain MRI parameters and other parameters at 5 and 10 years after the baseline evaluation. We will evaluate whether low muscle function (muscle mass, muscle strength or insulin sensitivity) is a risk factor for cognitive decline or cerebrovascular disease.
Strengths and limitations of this study

- Prospective cohort study over 10 years to identify risk factors for needing long-term care.

- Relationships between muscle mass, muscle strength or insulin sensitivity and multiple diseases necessitating long-term care will be evaluated simultaneously.

- A relatively large cohort was assembled for evaluating muscle mass, muscle strength, insulin sensitivity, whole brain MRI, cognitive function, vascular function, physical fitness and knee osteoarthritis, simultaneously.

- Study results may suggest novel strategies to prevent the need for long-term care.

- This study has several limitations: (1) including only participants living in an urban part of Japan, (2) challenges in confirming causal relationships and (3) a relatively low participants rate $(18.9 \%)$.

\section{INTRODUCTION}

The number of elderly people (aged $\geq 65$ years) has increased worldwide. For example, WHO defined the ageing rate as proportion of persons aged 65 years or older. The ageing rate has been increasing in both developed and low/middle-income countries. In 2015, the worldwide ageing rate was $8.3 \%$ and it is estimated to increase to $17.8 \%$ by 2055 . While the ageing rate in developed countries is predicted to reach $27.8 \%$ in $2060,{ }^{1}$ the ageing rate in Japan reached $27.7 \%$ in $2017^{2}$; it is the highest in the world. Japan is now categorised as a super-aged society, defined as a country with an ageing rate greater than $21 \% .^{34}$ The rate will reach $30 \%$ in 2025 and $40 \%$ in 2060 . A serious social problem in such a superaged society is the increase in the number of elderly people who need long-term care. 
Need for long-term care is defined as a condition where an elderly individual needs care to perform activities of daily living due to age-related diseases such as cerebrovascular disease, dementia and musculoskeletal disease. The number of individuals who need long-term care in Japan increased from 2.18 million in 2000 to 6.06 million in 2015. Social security expenses for long-term care increased from JP¥3.6 trillion in 2000 to JP¥10.1 trillion in $2015 .{ }^{56}$ These figures are predicted to increase and cause increased social and economic burden in Japan. Thus, the Japanese government has launched a policy to extend healthy life expectancy without long-term care ${ }^{7}$; however, evidence-based strategies to prevent the need for longterm care have not been established yet.

In Japan, the main causes of needing long-term care are cerebrovascular disease $(17.2 \%)$, dementia $(16.4 \%)$, age-related frailty $(13.9 \%)$, falls and fractures $(12.2 \%)$ and joint disease $(11.0 \%) .{ }^{2}$ Thus, multiple approaches to prevent these diseases will be useful for preventing the need for long-term care. However, if there are common risk factors for the onset of these diseases, it would be helpful to find an efficient strategy to prevent the need for long-term care. Several previous studies have demonstrated that a decrease in muscle mass or muscle strength $^{8-18}$ or insulin sensitivity ${ }^{19-25}$ may be common risk factors for diseases related to needing long-term care. Indeed, aging-related decreases in muscle strength or muscle mass ${ }^{8}$ are associated with an increased risk for metabolic syndrome, ${ }^{10-12} 26$ cognitive decline,${ }^{12} 14$ cerebrovascular disease ${ }^{27}{ }^{28}$ and all-cause mortality. ${ }^{13}{ }^{29}$ In addition, decreased muscle mass or muscle strength is also closely associated with decreased mobility, falls and fractures $^{15} 16$ as well as decreased psychosocial well-being ${ }^{17}$ and quality of life, ${ }^{18}$ which are associated with geriatric depression. On the other hand, impaired insulin sensitivity is associated with cardiovascular disease ${ }^{19} 20$ and lacunar infarcts ${ }^{2122}$ as well as microstructural white matter changes $^{23}$ and decreased cognitive performance. ${ }^{24} 25$
Given that skeletal muscle is the main organ that determines whole-body insulin sensitivity, ${ }^{30} 31$ we hypothesised that parameters related to muscle function such as muscle mass, muscle strength and insulin sensitivity might be the common risk factors for diseases related to needing longterm care. However, there is insufficient clinical evidence to support this hypothesis.

On the other hand, several other risk factors for diseases related to needing long-term care have been reported. For example, arteriosclerosis determined by CardioAnkle Vascular Index (CAVI) predicts cognitive decline in elderly Japanese individuals. ${ }^{32}$ A recent meta-analysis showed a modest association between CAVI and incident cardiovascular disease risk. ${ }^{33}$ In addition, cerebral small vessel disease (SVD), defined as lacunar infarcts, cerebral microhaemorrhages, periventricular hyperintensities (PVHs) or deep and subcortical white matter hyperintensities (DSWMHs) detected on MRI, is strongly associated with incident ischaemic and haemorrhagic stroke, all-cause dementia and depression, and all-cause mortality. ${ }^{34}$ Furthermore, a meta-analysis of data from 12 cohort studies showed that low hip bone mineral density (BMD) is an important predictor of fracture risk. ${ }^{35} \mathrm{We}$ hypothesised that muscle mass, muscle strength and insulin sensitivity are also common risk factors for arteriosclerosis, cerebral SVD and lower BMD; however, this hypothesis has not been tested yet.

In this context, we developed a prospective cohort study of elderly individuals in an urban community. The primary objective of the present study is to prospectively investigate associations between muscle mass, muscle strength or insulin sensitivity and main causes and risk factors for needing long-term care. The primary outcomes of the present study are incidence of cerebrovascular disease and cognitive decline. Secondary outcomes are incidence of age-related frailty, falls and fractures, and joint disease, respectively.

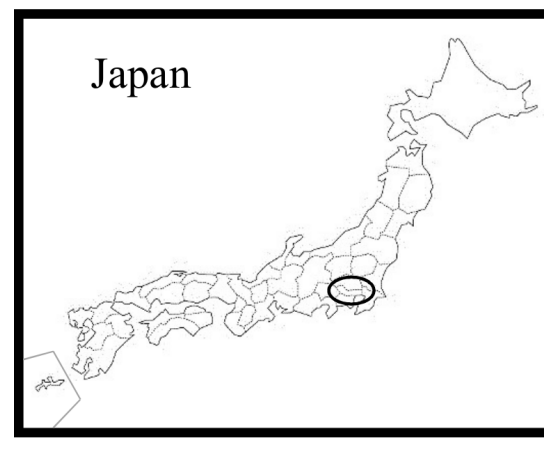

Japan is divided into 47
prefectures.

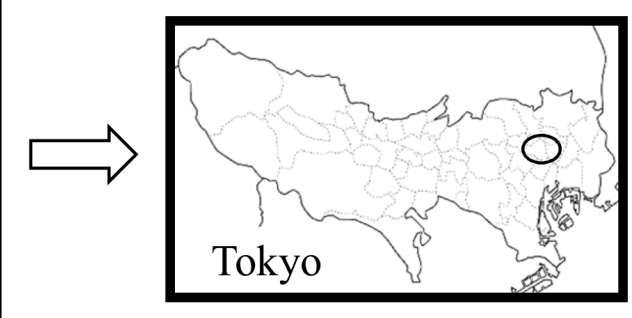

Tokyo is a prefecture that includes 23 special wards, 26 cities, 5 towns, and 8 villages.

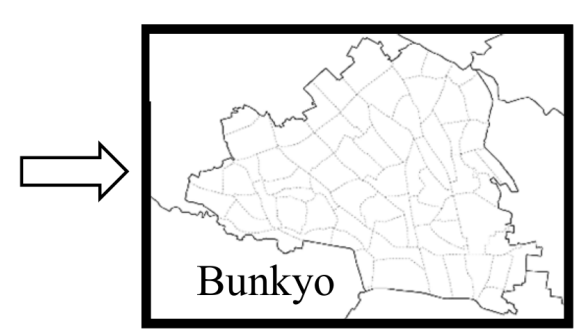

Bunkyo-ku is one of the special wards and includes 68 communities and 227,902 people.

Figure 1 Geographical location of Bunkyo-ku in Japan. Left: Japan is divided into 47 prefectures. The location of Tokyo is indicated by a circle. Centre: Tokyo is a prefecture that includes 23 special wards, 26 cities, 5 towns and 8 villages. Bunkyo is one of the special wards (Bunkyo-ku in Japanese). It located in east Tokyo, as indicated by the circle. Right: Bunkyo-ku includes 68 communities and has 227902 residents (source of map; http://www.start-point.net). 


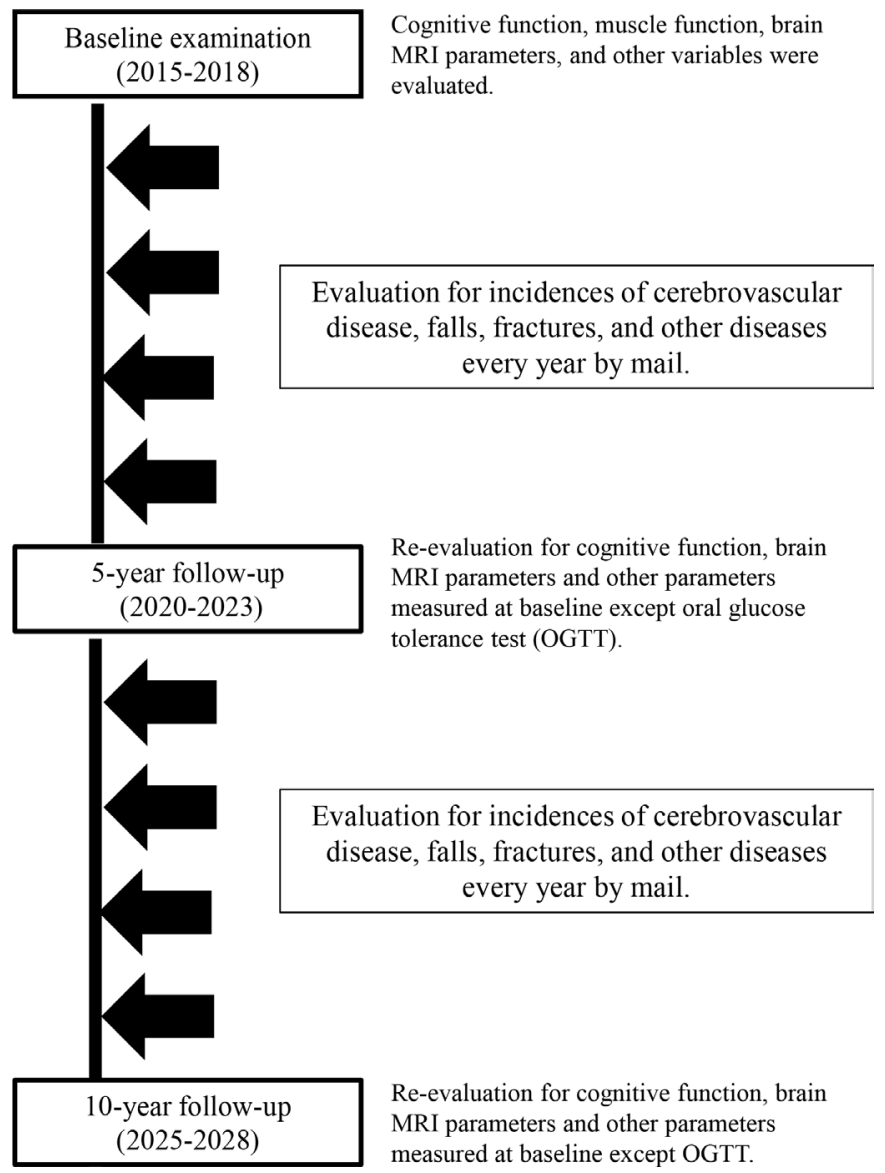

Figure 2 Experimental protocol.

\section{COHORT DESCRIPTION \\ Study design}

The Bunkyo Health Study is a prospective cohort study of over 10 years. We recruited elderly subjects aged 65-84 years living in Bunkyo-ku, an urban area in Tokyo, Japan (figure 1). Bunkyo-ku was selected because the age distribution in Bunkyo-ku is similar to that of Tokyo overall and there is high accessibility to the research centre. Among 68 communities in Bunkyo-ku, we selected 13 communities based on probability proportionate to size sampling. We obtained the name and address of all residents aged 65-84 years in the selected communities from residential registries. We mailed invitations to a group briefing session at our institution (Sportology Center, Juntendo University). The inclusion criterion of the present study was age of 65-84 years. The exclusion criteria were pacemaker or defibrillator placement and diabetes requiring insulin therapy. After the briefing session, subjects who provided written informed consent were included as research participants. The primary outcomes of the present study are incidence of cerebrovascular disease and cognitive decline. The secondary outcomes are age-related frailty, falls and fractures, and joint disease. The exploratory outcomes are arteriosclerosis, cerebral SVD and osteoporosis as well as cognitive function, brain volume and structure, depression, physical function and all-cause mortality.
The study protocol is outlined in figure 2. All subjects participated in examinations over two visits to the Sportology Center. During the first visit, subjects participated in cognitive function ${ }^{36} 37$ and physical fitness testing. ${ }^{38-42}$ MRI of the whole brain and the knee of the dominant leg or the leg with pain was performed. We also interviewed subjects about their medical history, family history and current pain. During the second visit, we evaluated body composition and BMD using dual-energy X-ray absorptiometry (DXA) ${ }^{43} \mathrm{CAVI}^{44}$ and abdominal fat distribution with MRI after an overnight fast. ${ }^{45}$ Next, we carried out a $75 \mathrm{~g}$ oral glucose tolerance test (OGTT) ${ }^{46}$ All data were collected and stored at the Sportology Center.

After the baseline evaluation (15 October 2015 to 1 October 2018), we will ask participants about their health status every year by mail. For example, we will ask about incidence of cerebrovascular disease, falls, fractures and other diseases every year. We also will send the Kihon Checklist questionnaire ${ }^{47}$ to participants every year. This questionnaire was developed to identify elderly individuals with frailty or at high risk for needing long-term care. It consists of 25 questions regarding instrumental and social activities of daily living, physical function, nutritional status, oral function, cognitive function and depressed mood. ${ }^{47} 48$ At 5 years $(2020-2023)$ and 10 years (2025-2028) after the baseline examination, we are planning to re-evaluate cognitive function, brain MRI parameters and other parameters measured at baseline except OGTT.

\section{Elderly subjects living in 13 communities of Bunkyo-ku $(n=8,629)$ \\ Excluded $(n=6,646)$ \\ -Unknown address: 242 \\ -Declined to participate: 292 \\ -Could not participate: 22 \\ -Did not reply: 6,090}

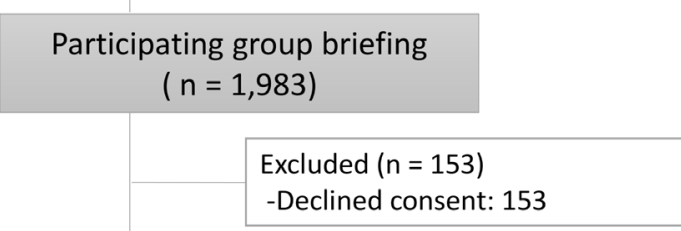

Informed consent obtained $(n=1,830)$

Excluded $(n=201)$

-Withdrew consent: 190

-Insulin treatment: 4

-Pacemaker implantation: 4

-Did not meet age criteria: 3

\section{This cohort subjects : 1,629}

Figure 3 Flow chart of the participant recruitment process. 


\section{Sample size calculations}

Since the primary outcomes are incidence of cerebrovascular disease and cognitive decline and the incidence of cognitive decline is lower than the incidence of cerebrovascular disease, ${ }^{49-51}$ the sample size was calculated to allow for evaluation of associations between cognitive decline and muscle mass, muscle strength and insulin sensitivity, respectively. Cognitive decline was defined as the presence of dementia or mild cognitive impairment (MCI) estimated using the Montreal Cognitive Assessment (MoCA), Japanese version ${ }^{36}$ or the MiniMental State Examination (MMSE), ${ }^{37} 53$ respectively. In the prospective analysis, we plan to categorise subjects in two groups for muscle function (eg, low and high muscle strength groups). The assumed incidence of cognitive decline per year is $1 \%-2 \%$ and the assumed cumulative incidence over 10 years in the population is $15 \% .^{5455}$ The low muscle function group was assumed to have a twofold higher risk of cognitive decline. Therefore, a sample of 1519 subjects was estimated to be necessary, assuming a drop-out rate of $15 \%$ over 10 years.

Since we also plan to perform a cross-sectional analysis using baseline data, we performed a preliminary sample size calculation. Although muscle mass, muscle strength and insulin sensitivity reflect different aspects of muscle function, the combined associations of these parameters with diseases or risk factors related to needing long-term care remain unclear. Thus, we plan to categorise subjects in nine categories of muscle function (eg, low, middle and high muscle strength $\times$ low, middle and high insulin sensitivity) and focus on associations between these categories of muscle function and cognitive decline. We also plan to evaluate other outcomes such as arteriosclerosis, cerebral SVD, osteoporosis, knee joint condition, cognitive function, brain volume and structure, depression, and physical function in the cross-sectional analysis. We estimated that rates of cognitive decline in the group with higher muscle function to be $12 \%$, compared with $24 \%$ in the group with lower muscle function. ${ }^{50} 51$ A sample of 1584 subjects was estimated to be necessary based on statistical power of $80 \%$ at a significance level of $5 \%$. Thus, the estimated required number of subjects in the cross-sectional analysis (1584) is sufficient for the prospective analysis. We set the overall sample size to be 1600 in the present study.

\section{Participants}

For the baseline examination (15 October 2015 to 1 October 2018), we mailed invitations to attend a group briefing session to all 8629 elderly individuals living in 13 communities in Bunkyo-ku, Tokyo, Japan, of whom $1984(23.0 \%)$ subjects participated in a group briefing session at our institution (Sportology Center, Juntendo University). After the briefing session, 1830 subjects provided written informed consent for study participation. However, 190 subjects withdrew consent and 11 subjects were excluded based on age, pacemaker status and insulin therapy status. Ultimately, the study subjects comprised 1629 elderly people, which corresponds to $18.9 \%$ of all elderly individuals in 13 selected communities of Bunkyo-ku) (figure 3).

\section{MEASUREMENTS}

\section{Muscle mass and strength}

Muscle mass was measured using DXA (Discovery DXA System, Hologic, Tokyo, Japan). ${ }^{56}$ Skeletal Muscle Mass Index (SMI) was calculated by dividing appendicular muscle mass $(\mathrm{kg})$ by height squared $\left(\mathrm{m}^{2}\right) .{ }^{57}$ Lower limb isokinetic muscle strength (angular velocity of $60^{\circ} / \mathrm{s}$ ) was measured using a dynamometer (BIODEX system 3 or 4: Biodex Medical Systems, Upton, New York, USA). ${ }^{58}$

\section{Insulin sensitivity}

Insulin sensitivity was estimated with the Matsuda index using data on glucose and insulin during the $75 \mathrm{~g} \mathrm{OGTT.}{ }^{46}$ The Matsuda index was calculated using the following equation: $10000 /$ square root of (fasting glucose $\mathrm{x}$ fasting insulin) $\mathrm{x}$ (mean glucose $\mathrm{x}$ mean insulin during OGTT). ${ }^{59}$ This formula can estimate whole-body glucose disposal during the euglycemic insulin clamp. Skeletal muscle is the main organ absorbing glucose during the clamp. ${ }^{30}$ Thus, the Matsuda index is a surrogate marker of insulin sensitivity, mainly reflecting insulin sensitivity in muscle. Our previous study using the two-step hyperinsulinemic euglycemic clamp technique demonstrated that the Matsuda index is significantly correlated with muscle insulin sensitivity, not hepatic insulin sensitivity. ${ }^{31}$

\section{Cognitive function}

Cognitive function was primarily evaluated using the MoCA $^{3652}$ and the MMSE. ${ }^{37}$ The MoCA and MMSE contain 9 and 11 items, respectively. Possible scores range from 0 to 30 points. In this study, we used MoCA score $\leq 22$ as the cut-off for MCI $^{6061}$ and MMSE score $\leq 23$ as the cut-off for dementia. ${ }^{3753}$ The Neurobehavioral Cognitive Status Examination, which evaluates orientation, attention, language, memory, calculation, construction and reasoning $^{6263}$ the Trail Making Test, which evaluates visual-conceptual and visual-motor tracking ${ }^{6465}$ and the Wechsler Memory Scale-Revised (part II) ${ }^{66}{ }^{67}$ were also used for evaluation of cognitive function. We also used the short version of the Geriatric Depression Scale ${ }^{6869}$ to assess depression, defined as a score $\geq 10$ points.

\section{Evaluation of whole brain MRI}

We performed whole brain MR scanning with a $0.3 \mathrm{~T}$ clinical MR scanner (AIRIS Vento, Hitachi, Tokyo, Japan) in all participants. In addition, brain MR scanning was also performed in some participants on a $3 \mathrm{~T}$ clinical MR scanner (MAGNETOM Prisma, Siemens Healthcare, Erlangen, Germany) with a 64-channel head coil for advanced brain MRI analysis and validation of brain analysis with the $0.3 \mathrm{~T}$ clinical MR scanner.

The following sequences were obtained with the 0.3 T clinical MR scanner: axial three-dimensional (3D) 
time-of-flight MR angiography (repetition time (TR), 35 $\mathrm{ms}$; echo time (TE), $7.1 \mathrm{~ms}$ and slice thickness, $1.2 \mathrm{~mm}$ ), T2*-weighted gradient echo imaging (TR, $1000 \mathrm{~ms}$; TE, $45 \mathrm{~ms}$; flip angle, $20^{\circ}$ and slice thickness, $5 \mathrm{~mm}$ ), and fluid-attenuated inversion recovery imaging (TR, $11000 \mathrm{~ms}$; TE, $100 \mathrm{~ms}$; inversion time (TI), $2000 \mathrm{~ms}$ and slice thickness, $5 \mathrm{~mm}$ ). We also obtained 3D-volumetric T1-weighted imaging using a gradient echo with inversion recovery sequence with these following parameters: TR, $25 \mathrm{~ms}$; TE, $5.8 \mathrm{~ms}$; TI, $600 \mathrm{~ms}$; flip angle, $12^{\circ}$; number of excitations, 1 ; field of view (FOV), $200 \times 250 \times 250 \mathrm{~mm}^{3}$; resolution, $0.98 \times 0.98 \times 2 \mathrm{~mm}^{3}$; slice orientation, sagittal; total scan time, $10.1 \mathrm{~min}$.

The protocol with the $3 \mathrm{~T}$ clinical MR scanner included 3D T1-weighted imaging and multishell diffusion weighted imaging (DWI). 3D T1-weighted images were obtained using magnetisation-prepared $180^{\circ}$ radio frequency pulses and rapid gradient-echo with these following parameters: TR, $2300 \mathrm{~ms}$; TE, $2.32 \mathrm{~ms}$; TI: 900 $\mathrm{ms}$; FOV, $240 \times 240 \mathrm{~mm}$; matrix size, 256 $\times 256$; resolution, $0.9 \times 0.9 \mathrm{~mm}$; slice thickness, $0.9 \mathrm{~mm}$ and acquisition time, $5.21 \mathrm{~min}$. For DWI, echo planar imaging (EPI) consisting of two b values ( 1000 and $2000 \mathrm{~s} / \mathrm{mm}^{2}$ ) along 64 isotropic diffusion gradients was acquired in the anterior-posterior phase-encoding direction with the following parameters: TR, $3300 \mathrm{~ms}$; TE, $70 \mathrm{~ms}$; FOV, $229 \times 229 \mathrm{~mm}$; matrix size, $130 \times 130$; resolution $1.8 \times 1.8 \mathrm{~mm}$; slice thickness, $1.8 \mathrm{~mm}$ and acquisition time, $7.29 \mathrm{~min}$. Each DWI acquisition was completed with a gradient-free image $(b=0)$. We also acquired standard and reverse phase encoded blipped images with no diffusion weighting (blip-up and blipdown) to correct for magnetic susceptibility-induced distortions related to EPI acquisitions.

Primary brain MRI evaluation was conducted by an experienced neuroradiologist based on axial $\mathrm{T} 2 *-\mathrm{WI}$ and FLAIR images obtained on a $0.3 \mathrm{~T}$ MR scanner. The evaluation included cerebral SVD, characterised by lacunar infarcts, cerebral microhaemorrhages, PVHs and DSWMHs. PVHs and DSWMHs were categorised using the Fazekas scale. ${ }^{70}$ In this study, level III was defined as cerebral SVD. In addition, whole brain volume, regional brain volume, regional cortical thickness, and white and grey matter integrity were also included on subsequent evaluations. Furthermore, $0.3 \mathrm{~T}$ and $3 \mathrm{~T}$ 3D T1-weighted images were used for the quantification of whole brain volume as well as regional cortical volume and thickness; multishell DWI was used for the quantification of white and grey matter integrity.

\section{Lifestyle and physical activity levels}

We investigated lifestyle and physical activity levels using questionnaires. To evaluate sleeping status, we used the Pittsburgh Sleep Quality Index, which has seven components. ${ }^{71} 72$ We evaluated exercise habits and physical activity levels with the International Physical Activity Questionnaire, which assesses different types of physical activity such as walking, moderate-intensity activities and vigorous-intensity activities. ${ }^{73}$ We evaluated nutritional status using a brief-type self-administered diet history questionnaire, which contains 58 items about fixed portions and food types. ${ }^{75}$ We evaluated mental status using the 12-Item Short Form Health Survey (Standard Japanese, Quality Metric, Lincoln, Rhode Island, USA) ${ }^{77}$ In addition, the Instrumental Activities of Daily Living scale was used to estimate the ability to live independently. ${ }^{78}$

\section{Physical fitness and function}

We evaluated physical fitness and function based on muscle strength with a hand grip dynamometer (T. K. K. 5401, Takei Scientific Instruments, Niigata, Japan) ${ }^{38}$ balance using the one-leg standing test, which measures the duration of one-leg standing with eyes open ${ }^{39}$; flexibility using the sit-and-reach test (T.K.K.5112, Takei Scientific Instruments) ${ }^{40}$; gait speed using the $10 \mathrm{~m}$ walking test $^{41}$ and combined motor function using the Timed Up and Go test, which measures the time it takes a participant to rise from an arm chair, walk $3 \mathrm{~m}$, turn, walk back and sit down again. ${ }^{42}$ In addition, locomotive functions were evaluated using the stand-up test, two-step test and a risk assessment on physical condition and lifestyle with 25 questions (Locomotive Challenge! Council, Japanese Orthopaedic Association, Tokyo, Japan).

\section{Blood and urine analysis}

Blood and urine samples were collected in the morning after an overnight fast. Subsequently, a standard $75 \mathrm{~g}$ OGTT was performed. Blood samples were obtained before and 30, 60, 90 and $120 \mathrm{~min}$ after ingesting $75 \mathrm{~g}$ of glucose to determine plasma glucose and serum insulin levels. Serum and plasma samples were sent to a commercial clinical laboratory (SRL, Tokyo, Japan). Biochemical analysis for each parameter in table 1 was performed using standard methods. Urine samples were used for albumin and qualitative tests. Other exploratory measurements are being planned for each study.

\begin{tabular}{ll}
\hline Table $1 \quad$ Parameters for blood and urine analyses \\
\hline Complete blood count & Phosphate \\
Glucose & Homocysteine \\
Insulin & Adiponectin \\
C-peptide & C reactive protein intact \\
HbA & parathyroid hormone \\
Triglyceride & 25-hydroxy vitamin D \\
High-density lipoprotein & Bone-specific alkaline \\
cholesterol & phosphatase \\
Low-density lipoprotein & Tartrate-resistant acid \\
cholesterol & phosphatase 5b \\
Free fatty acids & N-terminal probrain natriuretic \\
Aspartate & peptide \\
aminotransferase & Insulin-like growth factors-1 \\
Alanine aminotransferase & Dehydroepiandrosterone sulfate \\
$\gamma$-Glutamyl transferase & Free triiodothyronine \\
Albumin & Free thyroxine \\
Creatinine & Thyroid stimulating hormone \\
Calcium & Total testosterone \\
\hline
\end{tabular}


DNA extraction and genome-wide association study

Genomic DNA will be extracted from peripheral leucocytes. We plan to evaluate single nucleotide polymorphisms (SNPs) using an SNP array optimised for the Japanese or Asian population. ${ }^{79}$ DNA extraction and genome-wide association study will be performed to identify genes associated with clinical characteristics, mainly selected from the primary, secondary and exploratory outcomes.

\section{Bone mineral density}

BMD of the lumbar spine and femoral neck were measured using DXA (Discovery DXA System, Hologic). Quality assurance for longitudinal evaluation was performed by calibrating the machine with standardised phantoms. The coefficient of variation for the in vivo lumbar spine-BMD measurements was $<1 \%$. ${ }^{43}$ The diagnosis of osteoporosis was based on the criteria proposed by the Japanese Society of Bone and Mineral Metabolism in 2012. ${ }^{80}$ Postmenopausal women were diagnosed with osteoporosis if they met the following criteria: $\geq 1$ prevalent non-traumatic radiographic vertebral fractures based on clinical radiological criteria; hip fracture; low BMD $(\leq 70 \%$ of the young adult mean value); and BMD $\leq 80 \%$ of the young adult mean value and $\geq 1$ prevalent non-traumatic fragility fractures that are not vertebral or hip fractures.

\section{Arteriosclerosis}

Arteriosclerosis was estimated using CAVI and the Ankle Brachial Index (ABI), which were measured using an automatic waveform analyzer (Vascular Screening System VaSera VS-1500, Fukuda Denshi, Tokyo, Japan). ${ }^{44}$ Recording was performed in the supine position after 10 min of rest. Occlusion and monitoring cuffs were placed snugly on the upper and lower extremities. Pressure waveforms were then recorded simultaneously from the brachial arteries using the oscillometric method. All scans were automatically conducted by well-trained investigators. A resting $\mathrm{ABI} \leq 0.90$ was considered to reflect the presence of peripheral artery disease.

\section{Abdominal fat area}

Intra-abdominal fat area was measured with a $0.3 \mathrm{~T}$ MR scanner (AIRIS Vento, Hitachi). Subjects were in the prone position in the magnet with their arms placed straight overhead. Using the intervertebral space between the fourth and fifth lumbar vertebrae (L4-L5) as the point of origin, transverse images of $10 \mathrm{~mm}$ slice thickness were obtained every $100 \mathrm{~mm}$ from head to foot, resulting in a total of 10 images for each subject. All MRI data were transferred to a computer workstation for analysis using specialised image analysis software (AZE Virtual Place, AZE, Tokyo, Japan) to measure visceral and subcutaneous fat area.

\section{Anthropometric measurement}

Height was measured within $0.1 \mathrm{~cm}$ using a stadiometer in the upright position in the morning. Body weight was measured within $0.1 \mathrm{~kg}$ using an electronic scale (InBody770; Biospace, Seoul, Korea). Circumference of the waist as well as the thigh and calf of the dominant leg were measured within $0.5 \mathrm{~cm}$ with a plastic tape measure.
Evaluation of osteoarthritis of the knee joint and knee pain Radiographic OA severity ${ }^{81}$ was evaluated using the Kellgren-Lawrence grading system based on weight-bearing anteroposterior radiographs of the femorotibial joint for both knees in the bilateral standing extended view as well as weight-bearing posteroanterior radiographs of the femorotibial joint with the knee in $45^{\circ}$ of flexion.

Knee joints were also examined with MRI. Imaging sequences included coronal and sagittal proton density-weighted images with or without fat suppression and T2 mapping. Morphological changes in knee OA were semiquantitatively evaluated according to the Whole Organ MRI Score. ${ }^{82}$ Medial meniscus extrusion was also evaluated, we previously described ${ }^{83-86}$

Clinical manifestations of knee pain were evaluated using a Visual Analogue Scale of 0-100 mm and the Japanese Knee Osteoarthritis Measure (JKOM) score. ${ }^{87}$ JKOM is a self-reported score that includes four subcategories: pain and stiffness (0-32 points), activities of daily living ( $0-40$ points), social activities (0-20 points) and general health condition (0-8 points), with 100 points being the maximum score.

\section{Patient and public involvement}

No participants were involved in development of the research questions, design of the study or the recruitment.

\section{Statistical analysis}

Statistical analyses are planned to evaluate associations between muscle function and clinical outcomes. We use regression analysis with adjustment for age, sex and other potential factors, appropriately. Sex will be stratified as male and female. Age brackets will be 65-69, 70-74, 75-79 and $80-84$ years. The follow-up period is defined as the time from the baseline examination to the occurrence of each event during follow-up examinations. In addition, exploratory analyses will be performed with several exposures and outcomes. Statistical analysis plans will be appropriate to each study.

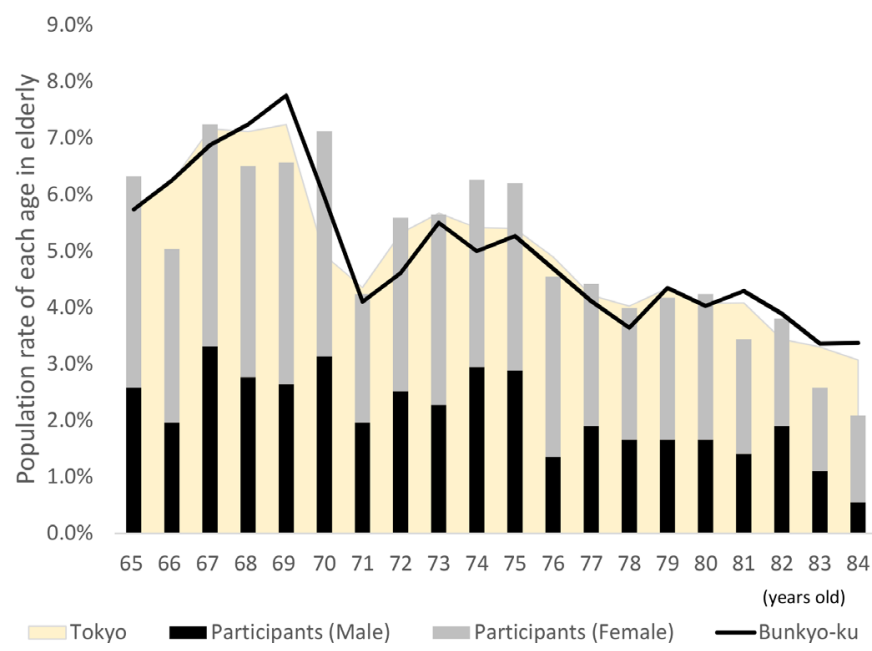

Figure 4 Distribution of the elderly population in this cohort, Bunkyo-ku and Tokyo. The proportion of elderly individuals in each age group (age 65-84 years) in this cohort, Bunkyo-ku and Tokyo are presented. 
Table 2 Characteristics of study subjects

N

Age (years old)

Height $(\mathrm{cm})$

Body weight $(\mathrm{kg})$

Body mass index $\left(\mathrm{kg} / \mathrm{m}^{2}\right)$

Education (year)

Worker/volunteer (n; \%)

Needing long-term care $(\mathrm{n} ; \%)$

Solitude (n; \%)

Hypertension ( $\mathrm{n} ; \%)$

Diabetes (n; \%)

Dyslipidemia (n; \%)

Cerebrovascular disease (n; \%)

Cardiovascular disease $(\mathrm{n} ; \%)$

Cancer (n; \%)

Cerebral small vessel disease $(\mathrm{n} ; \%)$

Systolic blood pressure $(\mathrm{mm} \mathrm{Hg})$

Diastolic blood pressure $(\mathrm{mm} \mathrm{Hg})$

Cardio Ankle Vascular Index: Right

Cardio Ankle Vascular Index: Left

Skeletal muscle mass $\left(\mathrm{kg} / \mathrm{m}^{2}\right)$

Muscle isokinetic strength $(\mathrm{Nm} / \mathrm{kg})$

Hang-grip strength $(\mathrm{kg})$

Gait speed (m/sec)

Timed up and Go test (sec)

Instrumental Activities of Daily Living (point)

Depression: GDS $\geq 10$ point (n; \%)

Fasting plasma glucose (mg/dL)

Fasting plasma insulin $(\mu \mathrm{U} / \mathrm{mL})$

$\mathrm{HbA} 1 \mathrm{c}(\%)$

Triglycerides (mg/dL)

HDL cholesterol ( $\mathrm{mg} / \mathrm{dL})$

LDL cholesterol (mg/dL)

Aspartate aminotransferase (IU/L)

Alanine aminotransferase (IU/L)

$\gamma$-glutamyl transferase (IU/L)

Serum albumin $(\mathrm{g} / \mathrm{dL})$

Creatinine $(\mathrm{mg} / \mathrm{dL})$

Matsuda index

Montreal Cognitive Assessment (MoCA) (point)

Mild Cognitive Impairment: MoCA $\leq 22$ point (n; \%)

Mini-Mental State Examination (MMSE) (point)

Dementia: MMSE $\leq 23$ point ( $\mathrm{n} ; \%)$

Bone mineral density of the femoral neck $\left(\mathrm{g} / \mathrm{cm}^{2}\right)$

Bone mineral density of the lumbar spine $\left(\mathrm{g} / \mathrm{cm}^{2}\right)$

\begin{tabular}{|c|c|c|}
\hline All participants & Male & Female \\
\hline 1629 & 687 & 942 \\
\hline $73.1 \pm 5.4$ & $73.0 \pm 5.3$ & $73.2 \pm 5.4$ \\
\hline $158.0 \pm 8.8$ & $165.8 \pm 5.9$ & $152.4 \pm 5.6$ \\
\hline $58.2 \pm 10.4$ & $65.7 \pm 8.6$ & $52.7 \pm 7.7$ \\
\hline $23.2 \pm 3.1$ & $23.9 \pm 2.8$ & $22.7 \pm 3.2$ \\
\hline $13.9 \pm 2.5$ & $14.9 \pm 2.5$ & $13.2 \pm 2.2$ \\
\hline 563 (34.6) & 288 (41.9) & 275 (29.2) \\
\hline $27(1.7)$ & $14(2.0)$ & $13(1.4)$ \\
\hline 342 (21.0) & 84 (12.2) & $258(27.4)$ \\
\hline 748 (45.9) & 368 (53.6) & $380(40.3)$ \\
\hline 187 (11.5) & $113(16.4)$ & $74(7.9)$ \\
\hline 639 (39.2) & 231 (33.6) & 408 (43.3) \\
\hline 68 (4.2) & 34 (4.9) & $34(3.6)$ \\
\hline $75(4.6)$ & $50(7.3)$ & $25(2.7)$ \\
\hline $40(2.5)$ & $25(3.6)$ & $15(1.6)$ \\
\hline $402(24.8)$ & $178(26.1)$ & 224 (23.9) \\
\hline $136.6 \pm 17.1$ & $136.8 \pm 16.4$ & $136.5 \pm 17.6$ \\
\hline $84.3 \pm 9.8$ & $86.4 \pm 9.7$ & $82.8 \pm 9.5$ \\
\hline $9.0 \pm 1.1$ & $9.2 \pm 1.1$ & $8.8 \pm 1.1$ \\
\hline $8.9 \pm 1.1$ & $9.1 \pm 1.0$ & $8.7 \pm 1.0$ \\
\hline $7.1 \pm 1.1$ & $7.9 \pm 0.9$ & $6.5 \pm 0.8$ \\
\hline $133.2 \pm 37.5$ & $148.4 \pm 38.8$ & $122.2 \pm 32.3$ \\
\hline $25.9 \pm 7.1$ & $32.3 \pm 5.7$ & $21.2 \pm 3.5$ \\
\hline $1.9 \pm 0.4$ & $2.0 \pm 0.4$ & $1.8 \pm 0.3$ \\
\hline $6.7 \pm 1.6$ & $6.5 \pm 1.6$ & $6.8 \pm 1.5$ \\
\hline $6.7 \pm 1.5$ & $5.0 \pm 0.3$ & $8.0 \pm 0.2$ \\
\hline $31(1.9)$ & $15(2.2)$ & $16(1.7)$ \\
\hline $100.5 \pm 16.7$ & $104.5 \pm 18.2$ & $97.6 \pm 14.8$ \\
\hline $4.9 \pm 3.3$ & $5.0 \pm 3.4$ & $4.8 \pm 3.2$ \\
\hline $5.8 \pm 0.6$ & $5.9 \pm 0.6$ & $5.8 \pm 0.5$ \\
\hline $98.5 \pm 54.2$ & $104.5 \pm 60.6$ & $94.1 \pm 48.6$ \\
\hline $64.3 \pm 16.5$ & $58.7 \pm 15.7$ & $68.4 \pm 16.0$ \\
\hline $121.3 \pm 30.8$ & $113.8 \pm 30.7$ & $126.8 \pm 29.7$ \\
\hline $23.5 \pm 9.5$ & $23.6 \pm 9.4$ & $23.4 \pm 9.6$ \\
\hline $19.3 \pm 11.1$ & $20.4 \pm 12.4$ & $18.5 \pm 10.1$ \\
\hline $30.8 \pm 35.8$ & $40.3 \pm 49.5$ & $23.8 \pm 17.8$ \\
\hline $4.3 \pm 0.4$ & $4.3 \pm 0.4$ & $4.3 \pm 0.4$ \\
\hline $0.8 \pm 0.3$ & $0.9 \pm 0.4$ & $0.7 \pm 0.2$ \\
\hline $7.3 \pm 4.1$ & $7.4 \pm 4.6$ & $7.2 \pm 3.7$ \\
\hline $25.1 \pm 3.0$ & $24.7 \pm 3.0$ & $25.5 \pm 3.0$ \\
\hline 295 (18.1) & $152(22.1)$ & $143(15.2)$ \\
\hline $27.7 \pm 1.9$ & $27.5 \pm 2.0$ & $27.9 \pm 1.9$ \\
\hline 53 (3.3) & 23 (3.3) & 30 (3.2) \\
\hline $0.6 \pm 0.1$ & $0.7 \pm 0.1$ & $0.6 \pm 0.1$ \\
\hline $0.9 \pm 0.2$ & $1.1 \pm 0.2$ & $0.8 \pm 0.2$ \\
\hline
\end{tabular}




\section{Findings to date}

The cohort was similar to the population of Bunkyo-ku and Tokyo, respectively (figure 4). This cohort characteristics was shown in table 2 . Average age was $73.1 \pm 5.4$ years (males: 73.0 \pm 5.3 , female: 73.2 \pm 5.4 ). The male:female ratio was $0.73: 1$ (males: $\mathrm{n}=687,42.2 \%$; females: $\mathrm{n}=942$, $57.8 \%$ ), which was also similar to that of Bunkyo-ku and Tokyo, respectively. The MoCA and MMSE scores for cognitive function were $25.1 \pm 3.0$ and $27.7 \pm 1.9$, respectively. Subjects with MCI (MoCA score $\leq 22$ ) and dementia (MMSE score $\leq 23$ ) represented $18.1 \%$ and $3.3 \%$ of the study cohort, respectively. The prevalence of MCI, dementia, hypertension and diabetes was comparable to the prevalence for those conditions in a similar elderly Japanese cohort. ${ }^{88-90}$ There were 402 participants (24.8\%) with cerebral SVD, which was also similar to the results of a previous study. ${ }^{49}$

In terms of muscle function, SMIs in males and females were $7.9 \pm 0.9 \mathrm{~kg} / \mathrm{m}^{2}$ and $6.5 \pm 0.8 \mathrm{~kg} / \mathrm{m}^{2}$, respectively. Muscle isokinetic strength was $148.4 \pm 38.8 \mathrm{Nm} / \mathrm{kg}$ in males and $122.2 \pm 32.3 \mathrm{Nm} / \mathrm{kg}$ in females. These findings are comparable to values in elderly Japanese subjects. ${ }^{88-90}$ Insulin sensitivity estimated using the Matsuda index was $7.3 \pm 4.1$, which is similar to the value in non-obese, non-diabetic and middle-aged men. ${ }^{31}$

\section{Strengths and limitations}

Strengths of the present study include: (1) long-term prospective cohort study design to identify risk factors for needing long-term care, (2) random sampling of elderly individuals in an urban area, (3) assembly of a relatively large cohort for evaluating muscle mass, muscle strength and insulin sensitivity, whole brain MRI, cognitive function, vascular function, physical fitness and knee OA simultaneously. Therefore, this study can evaluate relationships between muscle mass, muscle strength, and insulin sensitivity and multiple diseases that lead to needing long-term care. This study may suggest novel strategies to prevent the need for long-term care.

This study has several limitations such as (1) including only participants living in an urban part of Japan, (2) challenges in confirming causal relationships and (3) a relatively low participation rate $(18.9 \%)$, thus, we cannot deny the possibility of selection bias and we should be careful about the interpretation of the results. Before subject recruitment, we recognised that the participation rate in a similar study conducted in Japan was low $(18.4 \%) .{ }^{91}$ Thus, we sent invitation letters twice after no initial reply and called the subjects before the briefing session to increase the participation rate. Other plans may be considered to further increase the participation rate in future studies of community dwelling.

\section{Collaborators}

For more information or potential collaboration, please contact Yoshifumi Tamura (ys-tamur@juntendo.ac.jp) and Yuki Someya (yksomeya@juntendo.ac.jp).

\section{ETHICS AND DISSEMINATION}

All participants gave written informed consent at the orientation meeting. Participants were told that they have the right to withdraw from the trial at any time. Data are coded and stored securely in password-protected files. Only the principal investigators have access to the files. The findings of the study will be presented in local and international conferences and disseminated in peer-reviewed journals.

\section{Author affiliations}

${ }^{1}$ Sportology Center, Juntendo University Graduate School of Medicine, Tokyo, Japan ${ }^{2}$ Metabolism and Endocrinology, Juntendo University Graduate School of Medicine, Tokyo, Japan

${ }^{3}$ Juntendo University Graduate School of Health and Sports Science, Chiba, Japan

${ }^{4}$ Clinical Research Support Center, Juntendo University, Tokyo, Japan

${ }^{5}$ Cardiovascular Medicine, Juntendo University Graduate School of Medicine, Tokyo, Japan

${ }^{6}$ Medicine for Orthtopaedics and Motor Organ, Juntendo University Graduate School of Medicine, Tokyo, Japan

${ }^{7}$ Radiology, Juntendo University Graduate School of Medicine, Tokyo, Japan ${ }^{8}$ Clinical Laboratory Medicine, Juntendo University Graduate School of Medicine, Tokyo, Japan

${ }^{9}$ Neurology, Juntendo University Graduate School of Medicine, Tokyo, Japan ${ }^{10}$ Diagnosis, Prevention and Treatment of Dementia, Juntendo University Graduate School of Medicine, Tokyo, Japan

Contributors YS, YT, HK, HW and RK conceived the study and obtained grant funding. YS, YT and HK drafted the protocol. SN advised on the statistical analysis. KS, HD, MI, KK, TM, SH, SK, SA, NH, YM and HN reviewed and revised the protocol.

Funding This work is supported by the Strategic Research Foundation at Private Universities (S1411006) and KAKENHI (18H03184) from the Ministry of Education, Culture, Sports, Science and Technology of Japan, the Mizuno Sports Promotion Foundation, and the Mitsui Life Social Welfare Foundation.

Map disclaimer The depiction of boundaries on the map(s) in this article do not imply the expression of any opinion whatsoever on the part of BMJ (or any member of its group) concerning the legal status of any country, territory, jurisdiction or area or of its authorities. The map(s) are provided without any warranty of any kind, either express or implied.

Competing interests None declared.

Patient consent for publication Not required.

Ethics approval This study protocol was approved by the ethics committee of Juntendo University in November 2015 (no. 2015078, 2016138, 2016131 and 2017121). This study is being carried out in accordance with the principles outlined in the Declaration of Helsinki.

Provenance and peer review Not commissioned; externally peer reviewed. Data availability statement № data are available.

Open access This is an open access article distributed in accordance with the Creative Commons Attribution Non Commercial (CC BY-NC 4.0) license, which permits others to distribute, remix, adapt, build upon this work non-commercially, and license their derivative works on different terms, provided the original work is properly cited, appropriate credit is given, any changes made indicated, and the use is non-commercial. See: http://creativecommons.org/licenses/by-nc/4.0/.

\section{REFERENCES}

1. World population Prospects : The 2017 revision: United nations, 2017. Available: https://population.un.org/wpp/

2. Annual Report on the Aging Society 2018. Cabinet office, government of Japan, 2017. Available: http://www8.cao.go.jp/ kourei/whitepaper/w-2018/html/gaiyou/index.html [Accessed 27 Oct 2018].

3. Muramatsu N, Akiyama H. Japan: super-aging Society preparing for the future. Gerontologist 2011;51:425-32. 
4. Arai H, Ouchi Y, Toba K, et al. Japan as the front-runner of superaged societies: perspectives from medicine and medical care in Japan. Geriatr Gerontol Int 2015;15:673-87.

5. HealthandWelfareBureaufortheElderly. Long-Term care insurance system of Japan. MinistryofHealth, LabourandWelfare, 2016.

6. Tsutsui T. The current state and future development of the long-term care insurance system in Japan. Journal of the National Institute of Public Health 2010;59:372-9.

7. Kenkounippon21. Japan health promotion fitness Foundation, 2013. Available: http://www.kenkounippon21.gr.jp/

8. Rogers MA, Evans WJ. Changes in skeletal muscle with aging: effects of exercise training. Exerc Sport Sci Rev 1993;21:65-102.

9. Sanada K, Miyachi M, Tanimoto M, et al. A cross-sectional study of sarcopenia in Japanese men and women: reference values and association with cardiovascular risk factors. Eur J Appl Physiol 2010;110:57-65.

10. Zhang H, Lin S, Gao T, et al. Association between sarcopenia and metabolic syndrome in middle-aged and older non-obese adults: a systematic review and meta-analysis. Nutrients 2018;10:364.

11. Moon S-S. Low skeletal muscle mass is associated with insulin resistance, diabetes, and metabolic syndrome in the Korean population: the Korea National health and nutrition examination survey (KNHANES) 2009-2010. Endocr J 2014;61:61-70.

12. Yang EJ, Lim S, Lim J-Y, et al. Association between muscle strength and metabolic syndrome in older Korean men and women: the Korean longitudinal study on health and aging. Metabolism 2012;61:317-24.

13. LI RAN, XIA JIN, Zhang XI, Li R, Xia J, et al. Associations of muscle mass and strength with all-cause mortality among US older adults. Medicine \& Science in Sports \& Exercise 2018;50:458-67.

14. Kohara K, Okada $\mathrm{Y}$, Ochi M, et al. Muscle mass decline, arterial stiffness, white matter hyperintensity, and cognitive impairment: Japan Shimanami health promoting program study. J Cachexia Sarcopenia Muscle 2017;8:557-66.

15. Marcus RL, Addison O, Dibble LE, et al. Intramuscular adipose tissue, sarcopenia, and mobility function in older individuals. J Aging Res 2012;2012:1-6.

16. Ding L, Yang F. Muscle weakness is related to slip-initiated falls among community-dwelling older adults. J Biomech 2016;49:238-43.

17. Lee JSW, Auyeung T-W, Kwok T, et al. Associated factors and health impact of sarcopenia in older Chinese men and women: a crosssectional study. Gerontology 2007;53:404-10.

18. Tsekoura M, Kastrinis A, Katsoulaki M, et al. Sarcopenia and its impact on quality of life. Adv Exp Med Biol 2017;987:213-8.

19. Meigs JB, Rutter MK, Sullivan LM, et al. Impact of insulin resistance on risk of type 2 diabetes and cardiovascular disease in people with metabolic syndrome. Diabetes Care 2007;30:1219-25.

20. Krentz AJ, Viljoen A, Sinclair A. Insulin resistance: a risk marker for disease and disability in the older person. Diabetic Medicine 2013;30:535-48.

21. Lee JE, Shin DW, Yun JM, et al. Insulin resistance is a risk factor for silent lacunar infarction. Stroke 2016;47:2938-44.

22. Kaneda Y, Ishikawa S, Sadakane A, et al. Insulin resistance and risk of cerebral infarction in a Japanese general population: the Jichi medical school cohort study. Asia Pac J Public Health 2015;27:NP572-9.

23. Shimoji $\mathrm{K}, \mathrm{Abe} \mathrm{O}$, Uka $\mathrm{T}$, et al. White matter alteration in metabolic syndrome: diffusion tensor analysis. Diabetes Care 2013;36:696-700.

24. Lutski M, Weinstein G, Goldbourt U, et al. Insulin resistance and future cognitive performance and cognitive decline in elderly patients with cardiovascular disease. J Alzheimers Dis 2017;57:633-43.

25. Ekblad LL, Rinne JO, Puukka P, et al. Insulin resistance predicts cognitive decline: an 11-year follow-up of a nationally representative adult population sample. Diabetes Care 2017;40:751-8.

26. Sanada K, lemitsu M, Murakami H, et al. Adverse effects of coexistence of sarcopenia and metabolic syndrome in Japanese women. Eur J Clin Nutr 2012;66:1093-8.

27. Shimizu M, Misumi M, Yamada M, et al. Choice reaction time and grip strength as predictors of cardiovascular mortality in middle-aged and elderly Japanese: from the radiation effects research Foundation adult health study. Intern Med J 2018;48:1331-6.

28. Hirsch CH, Fried LP, Harris T, et al. Correlates of performance-based measures of muscle function in the elderly: the cardiovascular health study. J Gerontol A Biol Sci Med Sci 1997;52:M192-M200.

29. Landi F, Liperoti R, Fusco D, et al. Sarcopenia and mortality among older nursing home residents. J Am Med Dir Assoc 2012;13:121-6.

30. DeFronzo RA, Gunnarsson R, Björkman O, et al. Effects of insulin on peripheral and splanchnic glucose metabolism in noninsulindependent (type II) diabetes mellitus. J Clin Invest 1985;76:149-55.

31. Takeno K, Tamura Y, Kawaguchi M, et al. Relation Between Insulin Sensitivity and Metabolic Abnormalities in Japanese Men With BMI of $23-25 \mathrm{~kg} / \mathrm{m}^{2}$. The Journal of Clinical Endocrinology \& Metabolism 2016;101:3676-84

32. Yukutake T, Yamada M, Fukutani N, et al. Arterial stiffness predicts cognitive decline in Japanese community-dwelling elderly subjects: a one-year follow-up study. J Atheroscler Thromb 2015;22:637-44.

33. Matsushita K, Ding N, Kim ED, et al. Cardio-ankle vascular index and cardiovascular disease: systematic review and meta-analysis of prospective and cross-sectional studies. J Clin Hypertens 2019;21:16-24.

34. Rensma SP, van Sloten TT, Launer LJ, et al. Cerebral small vesse disease and risk of incident stroke, dementia and depression, and all-cause mortality: a systematic review and meta-analysis. Neuroscience \& Biobehavioral Reviews 2018;90:164-73.

35. Johnell O, Kanis JA, Oden A, et al. Predictive value of BMD for hip and other fractures. J Bone Miner Res 2005;20:1185-94.

36. Nasreddine ZS, Phillips NA, BÃ@dirian ValÃ@rie, Bedirian V, et al. The Montreal cognitive assessment, MoCA: a brief screening tool for mild cognitive impairment. J Am Geriatr Soc 2005;53:695-9.

37. Folstein MF, Folstein SE, McHugh PR, et al. "Mini-mental state". A practical method for grading the cognitive state of patients for the clinician. J Psychiatr Res 1975;12:189-98.

38. Bohannon RW. Muscle strength: clinical and prognostic value of hand-grip dynamometry. Curr Opin Clin Nutr Metab Care 2015;18:465-70.

39. Michikawa T, Nishiwaki Y, Takebayashi T, et al. One-leg standing test for elderly populations. Journal of Orthopaedic Science 2009;14:675-85.

40. Mayorga-Vega D, Merino-Marban R, Viciana J. Criterion-Related validity of Sit-and-Reach tests for estimating hamstring and lumbar extensibility: a meta-analysis. J Sports Sci Med 2014;13:1-14.

41. Peters DM, Fritz SL, Krotish DE. Assessing the reliability and validity of a shorter walk test compared with the 10-Meter walk test for measurements of gait speed in healthy, older adults. Journal of Geriatric Physical Therapy 2013;36:24-30.

42. Podsiadlo D, Richardson S. The timed "Up \& Go": a test of basic functional mobility for frail elderly persons. J Am Geriatr Soc 1991;39:142-8

43. McNamara EA, Kilim HP, Malabanan AO, et al. Enhanced precision of the new Hologic horizon model compared with the old discovery model is less evident when fewer vertebrae are included in the analysis. J Clin Densitom 2018;21:125-9.

44. Shirai K, Utino J, Otsuka K, et al. A novel blood pressureindependent arterial wall stiffness parameter; cardio-ankle vascular index (CAVI). J Atheroscler Thromb 2006;13:101-7.

45. Sato $\mathrm{F}$, Tamura $\mathrm{Y}$, Watada $\mathrm{H}$, et al. Effects of diet-induced moderate weight reduction on intrahepatic and intramyocellular triglycerides and glucose metabolism in obese subjects. J Clin Endocrinol Metab 2007;92:3326-9.

46. Kalyani RR, Metter EJ, Ramachandran R, et al. Glucose and insulin measurements from the oral glucose tolerance test and relationship to muscle mass. The Journals of Gerontology Series A: Biological Sciences and Medical Sciences 2012;67A:74-81.

47. Satake S, Senda K, Hong Y-J, et al. Validity of the Kihon checklist for assessing frailty status. Geriatr Gerontol Int 2016;16:709-15.

48. Arai H, Satake S. English translation of the Kihon checklist. Geriatr Gerontol Int 2015;15:518-9.

49. Fanning JP, Wong AA, Fraser JF. The epidemiology of silent brain infarction: a systematic review of population-based cohorts. BMC Med 2014;12.

50. Shimada H, Makizako H, Doi T, et al. Combined Prevalence of Frailty and Mild Cognitive Impairment in a Population of Elderly Japanese People. J Am Med Dir Assoc 2013;14:518-24.

51. Noguchi-Shinohara M, Yuki S, Dohmoto C, et al. Differences in the prevalence of dementia and mild cognitive impairment and cognitive functions between early and delayed responders in a communitybased study of the elderly. J Alzheimers Dis 2013;37:691-8.

52. Fujiwara $Y$, Suzuki $H$, Yasunaga $M$, et al. Brief screening tool for mild cognitive impairment in older Japanese: validation of the Japanese version of the Montreal cognitive assessment. Geriatr Gerontol Int 2010:10:225-32.

53. Ideno Y, Takayama M, Hayashi K, et al. Evaluation of a Japanese version of the Mini-Mental state examination in elderly persons. Geriatr Gerontol Int 2012;12:310-6.

54. Ohara T, Doi Y, Ninomiya T, et al. Glucose tolerance status and risk of dementia in the community: the Hisayama study. Neurology 2011;77:1126-34.

55. Shimada $\mathrm{H}$, Makizako $\mathrm{H}$, Doi $\mathrm{T}$, et al. Conversion and reversion rates in Japanese older people with mild cognitive impairment. J Am Med Dir Assoc 2017;18:808.e1-808.e6.

56. Bazzocchi A, Ponti F, Albisinni U, et al. DXA: technical aspects and application. Eur J Radiol 2016;85:1481-92. 
57. Chen L-K, Liu L-K, Woo J, et al. Sarcopenia in Asia: consensus report of the Asian Working group for sarcopenia. J Am Med Dir Assoc 2014;15:95-101.

58. Zawadzki J, Bober T, Siemienski A. Validity analysis of the Biodex system 3 dynamometer under static and isokinetic conditions. Acta Bioeng Biomech 2010;12:25-32.

59. Matsuda M, DeFronzo RA. Insulin sensitivity indices obtained from oral glucose tolerance testing: comparison with the euglycemic insulin clamp. Diabetes Care 1999;22:1462-70.

60. Luis CA, Keegan AP, Mullan M. Cross validation of the Montreal cognitive assessment in community dwelling older adults residing in the southeastern us. Int J Geriatr Psychiatry 2009;24:197-201.

61. Lee J-Y, Cho S-J, et al. Brief screening for mild cognitive impairment in elderly outpatient clinic: validation of the Korean version of the Montreal cognitive assessment. J Geriatr Psychiatry Neurol 2008;21:104-10.

62. Kiernan RJet al. The neurobehavioral cognitive status examination: a brief but differentiated approach to cognitive assessment. Ann Intern Med 1987;107:481-5.

63. Matsuda O, Nakatani M. Manual for Japanese version of the neurobehavioral cognitive status examination (COGNISTAT): world planning (in Japanese) 2014.

64. Reitan RM. The relation of the TRAIL making test to organic brain damage. J Consult Psychol 1955;19:393-4.

65. Hashimoto R, Meguro K, Lee E, et al. Effect of age and education on the TRAIL making test and determination of normative data for Japanese elderly people: the Tajiri project. Psychiatry Clin Neurosci 2006;60:422-8.

66. DA W. Wechsler memory scale-revised. New York: The Psychological Corporation. Harcourt Brace Jovanovich, Inc, 1987

67. Koike A, Sugishita M. [The Japanese version of the Wechsler Memory Scale--revised]. Nihon Rinsho 2011;69 Suppl 8(Suppl 8):408-12.

68. Sheikh JI, Yesavage JA, Scale GD. Gds): recent evidence and development of a shorter version. Clinical Gerontologist: The Journal of Aging and Mental Health 1986;5:165-73.

69. Sugishita K, Sugishita M, Hemmi I, et al. A validity and reliability study of the Japanese version of the geriatric depression scale 15 (GDS-15-J). Clin Gerontol 2017;40:233-40.

70. Fazekas F, Chawluk JB, Alavi A, et al. Mr signal abnormalities at 1.5 $\mathrm{T}$ in Alzheimer's dementia and normal aging. American Journal of Roentgenology 1987;149:351-6.

71. Doi Y, Minowa M, Uchiyama M, et al. Psychometric assessment of subjective sleep quality using the Japanese version of the Pittsburgh sleep quality index (PSQI-J) in psychiatric disordered and control subjects. Psychiatry Res 2000;97:165-72.

72. Buysse DJ, Reynolds CF, Monk TH, et al. The Pittsburgh sleep quality index: a new instrument for psychiatric practice and research. Psychiatry Res 1989;28:193-213.

73. Craig CL, Marshall AL, Sjostrom M, et al. International physical activity questionnaire: 12-country reliability and validity. Medicine \& Science in Sports \& Exercise 2003;35:1381-95.

74. Murase N, Katsumura T, Ueda U, et al. International standardization of physical activity level: reliability and validity study of the Japanese version of the International physical activity questionnaire (IPAQ). (Kosei no Shihyo) J Health Welfare Stat 2003;49:1-9.

75. Kobayashi S, Honda S, Murakami K, et al. Both comprehensive and brief self-administered diet history questionnaires satisfactorily
RANK nutrient intakes in Japanese adults. Journal of Epidemiology 2012;22:151-9.

76. Kobayashi S, Murakami K, Sasaki S, et al. Comparison of relative validity of food group intakes estimated by comprehensive and brief-type self-administered diet history questionnaires against $16 \mathrm{D}$ dietary records in Japanese adults. Public Health Nutr 2011:14:1200-11.

77. Ware J, Kosinski M, Keller SD. A 12-Item short-form health survey: construction of scales and preliminary tests of reliability and validity. Med Care 1996;34:220-33.

78. Lawton MP, Brody EM. Assessment of older people: selfmaintaining and instrumental activities of daily living. Gerontologist 1969;9:179-86.

79. Yamaguchi-Kabata Y, Nariai N, Kawai Y, et al. iJGVD: an integrative Japanese genome variation database based on whole-genome sequencing. Hum Genome Var 2015;2.

80. Soen S, Fukunaga M, Sugimoto T, et al. Diagnostic criteria for primary osteoporosis: year 2012 revision. J Bone Miner Metab 2013;31:247-57.

81. Kellgren JH, Lawrence JS. Radiological assessment of OsteoArthrosis. Ann Rheum Dis 1957;16:494-502.

82. Peterfy CG, Guermazi A, Zaim S, et al. Whole-Organ magnetic resonance imaging score (worms) of the knee in osteoarthritis. Osteoarthritis and Cartilage 2004;12:177-90.

83. Hada S, Kaneko H, Sadatsuki R, et al. The degeneration and destruction of femoral articular cartilage shows a greater degree of deterioration than that of the tibial and patellar articular cartilage in early stage knee osteoarthritis: a cross-sectional study. Osteoarthritis and Cartilage 2014;22:1583-9.

84. Hada S, Ishijima M, Kaneko H, et al. Association of medial meniscal extrusion with medial tibial osteophyte distance detected by T2 mapping $\mathrm{MRI}$ in patients with early-stage knee osteoarthritis. Arthritis Res Ther 2017;19.

85. Liu L, Ishijima M, Kaneko $\mathrm{H}$, et al. The MRI-detected osteophyte score is a predictor for undergoing joint replacement in patients with end-stage knee osteoarthritis. Modern Rheumatology 2017:27:332-8

86. Sadatsuki R, Ishijima M, Kaneko $\mathrm{H}$, et al. Bone marrow lesion is associated with disability for activities of daily living in patients with early stage knee osteoarthritis. J Bone Miner Metab 2019;37:529-36.

87. Akai M, Doi T, Fujino K, et al. An outcome measure for Japanese people with knee osteoarthritis. J Rheumatol 2005;32:1524-32.

88. The National health and nutrition survey: Ministry of health, labour and welfare, 2018. Available: http://www.nibiohn.go.jp/eiken/english/ research/project_nhns.html [Accessed 23 Jul 2018].

89. Makizako $\mathrm{H}$, Shimada $\mathrm{H}$, Tsutsumimoto $\mathrm{K}$, et al. Social frailty in community-dwelling older adults as a risk factor for disability. J Am Med Dir Assoc 2015;16:1003.e7-1003.e11.

90. Ryuno H, Kamide K, Gondo Y, et al. Longitudinal association of hypertension and diabetes mellitus with cognitive functioning in a general 70-year-old population: the sonic study. Hypertens Res 2017;40:665-70.

91. Monma T, Takeda F, Tsunoda K, et al. Age and gender differences in relationships between physical activity and sense of coherence in community-dwelling older adults. Jpn. J. Health Hum. Ecol 2015;81:159-69. 